\title{
GESTÃO DE REVISTA CIENTÍFICA ACADÊMICA: IMPLANTAÇÃO DE UM TEMPLATE NA REVISTA EDUCAÇÃO DA UNIVERSIDADE FEDERAL DE SANTA MARIA ${ }^{1}$
}

\author{
Clenio Perlin Berni ${ }^{2}$
}

ORCID: 0000-0002-1267-0357

Elena Maria Mallmann ${ }^{3}$

ORCID: 0000-0002-7611-3904

\begin{abstract}
Artigo elaborado a partir da dissertação de Mestrado.
Licenciado em Geografia, no Centro Universitário Franciscano (Unifra). Especialista em Gestores Regionais de Recursos Hídricos, Centro de Tecnologia da Universidade Federal de Santa Maria (UFSM). Atualmente é mestre pelo Programa de Pós-Graduação em Tecnologias Educacionais em Rede (UFSM). Atualmente exerce suas atividades administrativas, na área de Gestão de Revistas Científicas Acadêmicas e Editoração Científica, junto às Comissões Editorias das Revistas Educação (A1) e Educação Especial (A2) do Centro de Educação da UFSM. Tem experiência na área de editoração, com ênfase no Sistema Eletrônico de Editoração de Revistas (SEER), atuando principalmente nos seguintes temas: gestão de revistas científicas acadêmicas, editoração, tecnologias, Educação, Educação Especial. Contato: (clenioberni@gmail.com).
\end{abstract}

3 Graduação em Pedagogia e mestrado em Educação pela Universidade Federal de Santa Maria. Doutorado em Educação pela Universidade Federal de Santa Catarina. Pós-doutorado pela Universidade Aberta de Portugal - Bolsa Capes. Professora-pesquisadora do Departamento Administração Escolar (ADE). Orientadora de Mestrado e Doutorado no Programa de Pós-Graduação em Educação (PPGE) e no Programa de Mestrado Profissional em Tecnologias Educacionais em Rede (PPGTER). Coordenadora substituta do Programa de Pós-Graduação em Educação (PPGE) no biênio 2015-2017. Chefe Substituta do Departamento de Administração Escolar no biênio 2018-2010. Líder do Grupo de Pesquisa do CNPq: Grupo de Estudos e Pesquisas em Tecnologias Educacionais em Rede (GEPETER). Tem experiência na área de Educação, ênfase em Ensino-Aprendizagem/Tecnologia Educacional. Atua principalmente nas linhas de pesquisa Práticas Escolares e Políticas Públicas e Gestão de Tecnologias Educacionais em Rede nos temas: tecnologias educacionais, educação a distância, mediação pedagógica, Fluência Tecnológico-Pedagógica (FTP), materiais didáticos, Práticas Educacionais Abertas (PEA) e Recursos Educacionais Abertos (REA). Contato: (elena.ufsm@gmail.com). 
Resumo: Em um contexto de fácil ingresso e agilidade na troca de informações, deseja-se o aperfeiçoamento de ferramentas do sistema usado por periódicos, viabilizando o livre acesso à produção científica, permitindo a disseminação do conhecimento de forma mais dinâmica. Sendo assim, o problema central desse artigo é gerado a partir de reflexões acerca de como o acesso inicial para o envio de trabalhos pode ser otimizado e operacionalizado em plataforma eletrônica. Nesse sentido, a pesquisa objetivou a inserção de um template (modelo de padronização dos artigos originais recebidos) para o recebimento de artigos enviados ao periódico Educação (UFSM) do Centro de Educação da Universidade Federal de Santa Maria, via Sistema Eletrônico de Editoração de Revistas (SEER). Foi elaborado um template, que teve como propósito auxiliar a produção do texto pelos autores, padronizando o envio dos trabalhos. Após a implantação do template, foi utilizado como método de pesquisa, a aplicação de questionários junto aos autores e avaliadores ad hoc. $\mathrm{O}$ template implantado junto ao SEER, além de facilitar a interpretação das normas pelos autores, agilizou o processo editorial, uma vez que não será mais preciso retrabalhar os arquivos que chegam até o setor em diferentes formatações. Concluiu-se que a implantação do template foi eficaz para a revista, consolidando a gestão via portais institucionais, uma vez que não será mais necessário editorar os artigos na sua íntegra, fazendo com que os autores já digitalizem o seu artigo em campos específicos.

Palavras-chave: Editoração; Periódico; Template.

\title{
ACADEMIC JOURNAL MANAGEMENT: IMPLANTATION OF A TEMPLATE IN THE EDUCATION JOURNAL OF THE FEDERAL UNIVERSITY OF SANTA MARIA
}

\begin{abstract}
In a context of easy entry and agility in the exchange of information, it's desired to improve the tools of the system used by journals, enabling free access to scientific production, allowing the dissemination of knowledge more dynamically. Thus, the central problem of this article is generated from reflections about how the initial access for the sending of works can be optimized and operationalized in electronic platform. In this sense, the research aimed at the insertion of a template (standardization model of the original articles received) for the reception of articles sent to the Education (UFSM) journal of the Education Center of the Federal University of Santa Maria, via the Electronic Publishing System (SEER). A template was developed, whose purpose was to help the authors produce the text, standardizing the submission of papers. After the implantation of the template, the questionnaire was applied as a research method to authors and ad hoc evaluators. The SEER template, in addition to facilitating the interpretation of the standards by the authors, expedited the editorial process, since it will no longer be necessary to rework the files that arrive to the sector in different formats. It was concluded that the implementation of the template was effective for the journal, consolidating the management via institutional portals, since it will no longer be necessary to publish the articles in their entirety, causing the authors to digitize their article in specific fields.
\end{abstract}

Keywords: Publishing; Newspaper; Template. 


\section{GESTIÓN DE REVISTA CIENTÍFICA ACADÉMICA: IMPLANTACIÓN DE UN TEMPLATE EN LA REVISTA EDUCACIÓN DE LA UNIVERSIDAD FEDERAL DE SANTA MARÍA}

Resumen: En un contexto de fácil ingreso y agilidad en el intercambio de informaciones, se desea el perfeccionamiento de herramientas del sistema usado por periódicos, viabilizando el libre acceso a la producción científica, permitiendo la diseminación del conocimiento de forma más dinámica. Siendo así, el problema central de este artículo es generado a partir de reflexiones acerca de cómo el acceso inicial para el envío de trabajos puede ser optimizado y operacionalizado en plataforma electrónica. En este sentido, la investigación objetivó la inserción de un template (modelo de estandarización de los artículos originales recibidos) para la recepción de artículos enviados al periódico Educación (UFSM) del Centro de Educación de la Universidad Federal de Santa María, vía Sistema Electrónico de Edición de Revistas (SEER). Se elaboró un template, que tuvo como propósito auxiliar la producción del texto por los autores, estandarizando el envío de los trabajos. Después de la implantación del template, fue utilizado como método de investigación, la aplicación de cuestionarios junto a los autores y evaluadores ad hoc. El template implantado junto al SEER, además de facilitar la interpretación de las normas por los autores, agilizó el proceso editorial, ya que ya no será necesario retrabajar los archivos que llegan hasta el sector en diferentes formatos. Se concluyó que la implantación del template fue eficaz para la revista, consolidando la gestión vía portales institucionales, ya que ya no será necesario publicar los artículos en su totalidad, haciendo que los autores ya digitalizan su artículo en campos específicos.

Palabras claves: Publishing; Diario; Template.

Submetido em: 22/05/2019.

Aceito em: 01/08/2019.

\section{INTRODUÇÃO}

A pesquisa desenvolvida neste trabalho constituiu-se pela necessidade de analisar como a implantação de um template ${ }^{4}$, inserido junto às ferramentas do Sistema Eletrônico de Editoração de Revistas (SEER) do Portal de Periódicos Eletrônicos da Universidade Federal de Santa Maria (UFSM), para o recebimento de artigos originais submetidos à revista Educação (UFSM), facilitou a compreensão das normas pelos autores e agilizou na editoração final do periódico

4 Template ou "modelo de documento" é um documento de conteúdo, com apenas a apresentação visual (apenas cabeçalhos, por exemplo) e instruções sobre onde e qual tipo de conteúdo deve entrar a cada parcela da apresentação (por exemplo, conteúdos que podem aparecer no início e conteúdos que só podem aparecer no final). WIKIPÉDIA. Web template. [S.I.], 2017. Disponível em: https:// pt.wikipedia.org/wiki/Web_template. Acesso em: 10 jan. 2018. 
Educação (UFSM) do Centro de Educação (CE) da UFSM. Utiliza-se o SEER, desde 2008, baseado no movimento de Acesso Livre à Informação Científica. Desse modo, agrega-se facilidades aos usuários (autores, leitores, editores, avaliadores e demais usuários de portais institucionais educacionais) para uma gestão-ação do conhecimento. Portanto, a meta trabalhada é para que não seja necessário editorar os artigos na sua íntegra e, assim como os indexadores ${ }^{5}$, fazer com que os autores já digitalizem o seu artigo em campos específicos. Nesse sentido, o template é um dispositivo inicial para a transformação pretendida.

\section{O PERIÓDICO CIENTÍFICO ELETRÔNICO}

A produção científica disponibilizada em periódicos vem se consolidando como a base para o desenvolvimento científico e tecnológico. As transformações ocorridas com o avanço das Tecnologias de Informação e Comunicação (TIC) e o uso de software não proprietário e proprietário, voltados para a editoração de periódicos, facilitaram o acesso à informação científica e possibilitaram o surgimento de novas alternativas para a difusão da ciência. Nesse sentido, a pesquisa buscou, através da inserção do template no sistema, facilitar a compreensão das normas pelos autores, bem como aprimorar e agilizar a atividade editorial do periódico. Visa-se atender às exigências de um portal institucional e dos indexadores internacionais de periódicos (foco e missão da revista) sob a gestão da Comissão Editorial da Revista Educação (UFSM) do CE/UFSM. Nesse contexto de fácil ingresso e agilidade na troca de informações, deseja-se o aperfeiçoamento de ferramentas do sistema usado pelo periódico, viabilizando o livre acesso à produção científica, permitindo a disseminação do conhecimento de forma mais dinâmica. Dessa maneira, objetiva-se tornar mais visível a produção acadêmica de diversas áreas do conhecimento, inseridas nos textos da revista científica editada pelo Laboratório de Pesquisa e Documentação em Educação (LAPEDOC) e perpetuadas no portal institucional da UFSM.

Atualmente, pode-se considerar que a utilização das TIC já está incorporada a praticamente toda a cadeia de editoração científica para revistas eletrônicas. Algumas exceções de periódicos ainda publicam em formato impresso em conjunto com o modo online, mas a tendência é que, gradativamente, venham a desaparecer as produções em papel (em virtude do alto custo da publicação impressa

5 Os indexadores reúnem um conjunto de títulos de periódicos que passaram por um processo de seleção. 
e da pouca procura pelos acadêmicos, que veem nas tecnologias, em especial as móveis, facilidade e rapidez no acesso, sem precisar deslocar-se do seu local de estudos ou residência para ter acesso aos conteúdos científicos produzidos, bem como pela facilidade de buscar somente o assunto que se deseja trabalhar). A evolução na gestão e aplicabilidade das tecnologias educacionais e que, preferencialmente, utilizam software não proprietário, vindo ao encontro dos processos de editoração de periódicos eletrônicos das instituições, principalmente as públicas, pode ser observada na afirmação de Oliveira (2008):

As modificações ocorridas nos processos de comunicação científica nos últimos anos trazem a necessidade de uma nova forma de organização dos processos de trabalho organizacional e de informação. Características como interatividade, compartilhamento e distribuição da informação estão presentes nos meios eletrônicos. Assim, questões como a garantia de autoria e de direitos autorais, permanência e validade da informação e políticas de acesso por parte de editoras, devem ser discutidas e definidas para que a comunicação, através do ambiente eletrônico que utilizam software livre, possam ser plenamente aceitos pela comunidade científica (OLIVEIRA, 2008, p. 69).

No contexto educacional brasileiro atual, em relação ao processo exercido na editoração de periódicos institucionais com qualidade e credibilidade para divulgação da ciência produzida na universidade, o processo editorial de periódicos apresenta-se como um mecanismo institucional importante. Está diretamente relacionado à cadeia produtiva do desenvolver conhecimento e disponibilizá-lo à comunidade, através dos mecanismos tecnológicos digitais capazes de armazenar e distribuir conteúdo com validade científica. A revista Educação (UFSM) busca a difusão do conhecimento e contribui para a credibilidade que os portais institucionais vêm conquistando perante a comunidade científica. Possui ideais baseados na produção de conhecimento, de forma a disponibilizar à população conteúdo científico de qualidade (avaliado por pares $^{6}$ ), de fácil acesso e que atenda ao maior número possível de cidadãos.

Sobre esse tema, a afirmação que mais coaduna com os propósitos de um portal institucional é a definição de autores como Oliveira (2008):

Entende-se por periódico eletrônico aquela publicação que pretende ser continuada indefinidamente, que apresente procedimentos de controle de qualidade dos trabalhos publicados aceitos internacionalmente, e que disponibilize o texto completo do artigo através de acesso online, podendo ter ou não uma versão impressa ou em outro tipo de suporte (OLIVEIRA, 2008, p. 71).

6 A avaliação por pares significa que o artigo será analisado por dois avaliadores distintos. 
O problema central deste trabalho foi gerado a partir de reflexões acerca de como o acesso inicial para o envio de trabalhos podia ser otimizado e operacionalizado em plataforma eletrônica. Em outras palavras, em que medida a reorganização do modo de padronizar um artigo para ser enviado ao periódico Educação (UFSM), o qual é gerenciado pelo LAPEDOC, contribui para a melhoria do trabalho editorial em suas etapas?

A pesquisa teve como enfoque a revista Educação (UFSM), hospedada no Portal de Periódicos Eletrônicos da UFSM, na qual foi realizado experimento prático da investigação para customizar o acesso virtual e digital para dispositivos eletrônicos. Os resultados da pesquisa foram validados no trabalho diário de verificação e/ou aprovação dos artigos recebidos para análise junto à comissão editorial do periódico. Dessa maneira, esta pesquisa tratou-se de um trabalho de investigação, análise e proposição à Revista do CE, gerenciada pelo LAPEDOC, na inserção de uma template para recebimento dos artigos junto ao SEER.

O objetivo geral constituiu-se pela necessidade de analisar como a implantação de um template, inserido junto às ferramentas do SEER do Portal de Periódicos da UFSM, para o recebimento dos artigos originais submetidos à revista Educação (UFSM), facilitou a compreensão das normas pelos autores e agilizou na editoração final do periódico. Como objetivos específicos implementou-se um template junto à revista Educação (UFSM), baseado nas normas de recebimento dos artigos a serem avaliados pelo periódico; e avaliou-se a pós-implantação do modelo para recebimento dos artigos, com base nas respostas obtidas no questionário, constatando se houve ou não melhorias e facilidades aos autores e avaliadores ad hoc do periódico.

O portal tornou-se o meio de disseminação científica mais eficaz e ágil, pelo qual a ação conjunta de autores, editores e avaliadores tem o controle científico e institucional da qualidade e, consequentemente, da confiabilidade do conhecimento disponibilizado pelos editores à sociedade. Para Valerio (2005), a combinação dos mecanismos de controle de qualidade dá validade à ciência que, por sua vez, só adquire tal condição quando se torna pública.

\section{O ACESSO AO PORTAL DE PERIÓDICOS POR DISPOSITIVOS ELETRÔNICOS}

A partir da implantação dos Portais nas universidades, principalmente nas públicas, foi possível proporcionar maiores oportunidades para estudantes de todas as instituições de ensino e classes sociais, especialmente as menos favorecidas. Com isso, essas instituições agora têm alcance a conteúdo publicado em periódicos 
reconhecidos, um material de qualidade com acesso gratuito, o que antes só era disponível por assinatura junto às editoras privadas, que têm em sua plataforma de governo o lucro para as empresas e não a disseminação do conhecimento à população.

Assim, desde finais do século XX, trabalha-se em um processo de transição, pois antes os arquivos de artigos originais precisavam ser impressos, sendo a única maneira que as pessoas podiam ter acesso aos materiais publicados pelas editoras ou universidades, os quais eram disponibilizados principalmente em bibliotecas, livros ou periódicos. Como afirma Maia (2005, p. 15): "Vivencia-se uma transição das atividades relacionadas com a manipulação, a edição, o armazenamento, a distribuição e a recuperação da informação da forma impressa para a forma digital [...]". Os acadêmicos e pesquisadores, interessados no aprimoramento de seu conhecimento, precisavam de cópias físicas para buscar as informações. Hoje, porém, quase tudo se encontra de forma online. Com essa evolução digital, tornou-se essencial desenvolver mecanismos capazes de suportar e propagar conteúdo de forma ágil no modelo eletrônico, para receber, produzir e armazenar a informação, seja ela em dispositivo fixo ou portátil, e que ultrapasse o meio físico da impressão.

As ferramentas tecnológicas, voltadas a produzir ciência, possuem, na sua estrutura, elementos que podem ajudar na sistematização editorial de novos produtos a serem inseridos em um programa tecnológico de divulgação, alargando o horizonte e proporcionando um ritmo acelerado no compartilhar conhecimento através das organizações, no caso, o portal institucional. Também identifica, desenvolve e implanta ferramentas tecnológicas que apoiem à comunicação institucional na gestão e aplicabilidade de programas voltados à produção de novos conceitos e conteúdos. As ferramentas tecnológicas têm papel importante no mecanismo da infraestrutura, mas é o aspecto humano envolvido no gerenciamento dessas ferramentas e na produção de novos conhecimentos a peça chave para a boa gestão institucional de partilhar conhecimento, com participação coletiva das pessoas.

\section{O PRODUTO - TEMPLATE}

O produto apresentado ao periódico Educação (UFSM), junto ao SEER do Portal da UFSM, trouxe, já no recebimento dos artigos, uma melhor qualidade, visto que é uma forma de orientação ao autor. Ele permite a quem submete as produções conhecer quais procedimentos deve seguir a fim de que seu trabalho seja acolhido pelo periódico, para só então estar apto a análise por parte da Comissão encarregada. A comissão editorial da Revista, e também do Portal de Periódicos da Instituição, trabalha com o objetivo de mostrar o seu produto, ou 
seja, o periódico, visando divulgar, compartilhar, coletar, junto aos seus usuários, colaborações no sentido de somar e aperfeiçoar o sistema vigente. Visto que, em se tratando de ferramentas tecnológicas, sempre é possível melhorar, principalmente quando várias pessoas pensam juntas com um mesmo propósito: o de somar para continuar crescendo. Assim, caminha-se na busca incessante da credibilidade do periódico, conquistada com o compartilhar de todos envolvidos no processo de gerar ciência dentro das universidades, principalmente as públicas.

A implementação do template começou a ganhar força quando autores começaram a solicitar, através da comunicação via sistema SEER, antes do envio de suas produções, se a revista possuía um modelo para o artigo a ser inserido no sistema. Perante essa solicitação, fez-se uma busca em outros periódicos de portais e constatou-se que algumas das revistas pesquisadas, as mais conhecidas do país, já dispunham desse mecanismo. Então, pesquisados os dados, levou-se ao conhecimento da Comissão a intenção de implementar um template para o periódico Educação (UFSM) (Figura 1). Após alguns esclarecimentos e uma breve demonstração do funcionamento aos membros da Comissão, a ideia foi aceita, sendo aprovada sua implantação junto ao sistema SEER. O template da revista Educação (UFSM) foi desenvolvido com base nas normas NBR 6022, NBR 6023, NBR 6024, NBR 6028, NBR 10520 e NBR 10525 da Associação Brasileira de Normas Técnicas (ABNT).

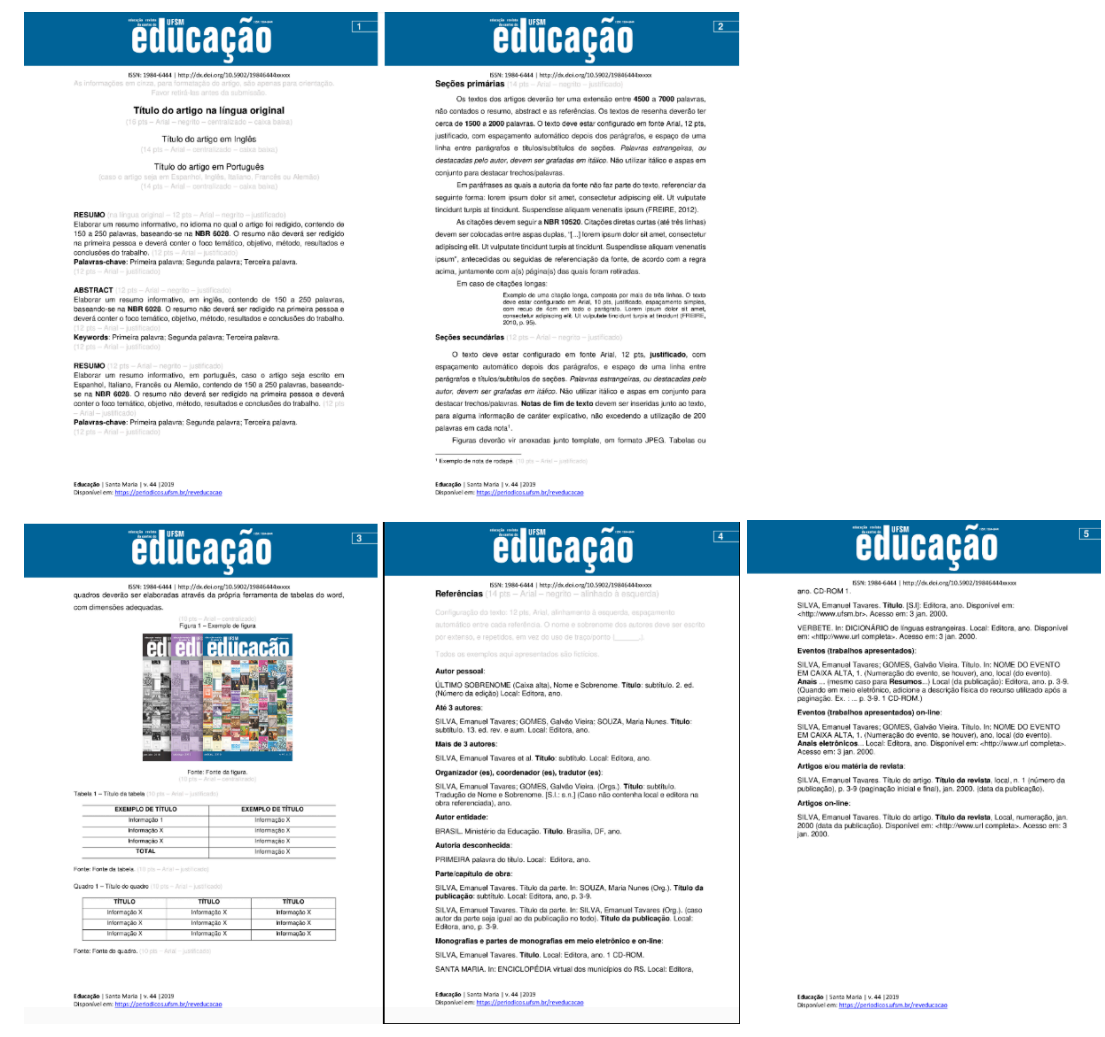

Figura 1: Modelo de template da ReVista Educação (UFSM). Fonte: Site da ReVista. 


\section{A PESQUISA - REVISTA EDUCAÇÃO (UFSM)}

A revista Educação (UFSM) foi criada em 1970 e, em 1991, passou a denominar-se Educação (UFSM). De 1970 a 2007, era publicada semestralmente e, em 2008, passou para quadrimestral, funcionando assim até o ano de 2017. O periódico passou por mudanças significativas em seu escopo e na forma de editoração, uma vez que, em 2008, foi implantado o sistema SEER para todo o fluxo editorial, desde o recebimento das colaborações até a sua publicação. Nova alteração aconteceu em 2018, quando a revista passou a ser editada trimestralmente. A revista Educação (UFSM) tem sido publicada de forma ininterrupta e busca sempre a pontualidade, tendo como diferencial a publicação da edição já no primeiro mês de cada trimestre. Assim, itens como caráter científico, avaliação por pares, conselho editorial, periodicidade, duração, pontualidade, resumo, palavras-chave e título em inglês, normalização, afiliação de autores e procedimentos do processo de admissão já são práticas incorporadas pelo Conselho Editorial na editoração da Revista. Constantemente, projetam-se ações de melhorias para a construção do periódico, buscando sua maior visibilidade acadêmica na área de Educação em nível internacional. A Revista percorreu um longo caminho e cresceu muito, sendo hoje uma referência no contexto das produções acadêmicas de sua área, obtendo o conceito A1 do Qualis ${ }^{7} / \mathrm{CAPES}^{8}$.

Hoje, com 48 anos de existência, pode-se dizer que a revista Educação (UFSM) teve uma trajetória vitoriosa, visto que é notório o seu crescimento e reconhecimento internacional, pois conta com indexadores renomados no meio institucional e acadêmico, tanto do país como do exterior. A Revista está disponível na forma digital desde 2000, porém, o processo do todo fluxo editorial, via SEER, foi implantado a partir de 2008, garantindo maior agilidade na troca das informações entre autores, editores e avaliadores, permitindo maior visibilidade e credibilidade perante a comunidade científica.

A pesquisa amparou-se nos postulados teórico-metodológicos da pesquisa-ação e está diretamente ligada à etapa de recebimento dos artigos para a revista Educação (UFSM), na qual implementou-se o template no sistema SEER. O modelo empreendido serviu como um dispositivo facilitador aos autores, para que o trabalho não chegue ao periódico fora das normas estabelecidas, o que é procedimento padrão e obrigatório para sua tramitação. Aplicou-se um questionário

\footnotetext{
Conjunto de procedimentos utilizados pela CAPES para estratificação da qualidade da produção intelectual dos programas de pós-graduação. Disponível em: http://www.capes.gov.br/component/content/ article?id=2550:capes-aprova-a-nova-classificacao-do-qualis. Acesso em: 19 jul. 2018.

8 Coordenação de Aperfeiçoamento de Pessoal de Nível Superior.
} 
junto aos autores e avaliadores ad hoc, em que foi perguntado sobre a avaliação dos mesmos após a implementação do template para o recebimento de artigos.

A coleta de dados serve para definir a direção que um trabalho seguirá através das informações apanhadas, as quais são de suma importância, pois são dados levantados a partir da participação do público-alvo, chamados de colaboradores. Assim, na caminhada do trabalho de edição de um periódico, ocorre a análise da eficácia, entre outros pontos, que dizem respeito ao dispositivo implantado no sistema (e agora investigado). Tem como objetivo reunir um conjunto de informações que comprovem determinada ação metodológica. Na pesquisa realizada, junto ao sistema eletrônico, objetivou-se respostas positivas para o desenvolvimento das ações editoriais do trabalho do setor. Está fundamentada através dos dados coletados por meio da pesquisa no sistema e do questionário aplicado, que dão base para o desenvolvimento da pesquisa.

A tabela a seguir (Tabela 1) demonstra o fluxo dos artigos recebidos pela revista Educação (UFSM), no período de janeiro de 2018 a agosto de 2018, quando se implantou o dispositivo template no sistema SEER para o recebimento dos trabalhos dos respectivos autores.

\begin{tabular}{|l|c|c|c|c|c|c|c|c|c|}
\hline \multicolumn{1}{|c|}{ StAtus dos ARTIGos } & JAN & FEv & MAR & AB & MAI & JUN & JUL & AGo & TOTAL \\
\hline Artigos recebidos & 73 & 68 & 74 & 70 & 62 & 81 & 67 & 67 & 562 \\
\hline $\begin{array}{l}\text { Recebidos adequados } \\
\text { ao template }\end{array}$ & 18 & 09 & 24 & 35 & 32 & 47 & 27 & 31 & 223 \\
\hline $\begin{array}{l}\text { Devolução/Sem } \\
\text { template }\end{array}$ & 25 & 28 & 23 & 17 & 17 & 27 & 22 & 13 & 172 \\
\hline $\begin{array}{l}\text { Template adequado } \\
\text { que retornaram }\end{array}$ & 15 & 22 & 13 & 10 & 10 & 16 & 12 & 6 & 104 \\
\hline $\begin{array}{l}\text { Devolução/ } \\
\text { Fora do escopo }\end{array}$ & 30 & 31 & 27 & 18 & 13 & 7 & 18 & 23 & 167 \\
\hline Devolução/Total geral & 55 & 59 & 50 & 35 & 30 & 34 & 40 & 36 & 339 \\
\hline
\end{tabular}

TABELA 1: FluXo EDITORIAL DOS ARTIGOS NO PERÍOdO DE JANEIRO DE 2018 A ACOSTO DE 2018 DA REVISTA EdUCAÇÃo (UFSM). FONTE: ELABORADA PELOS AUTORES (2018) ${ }^{9}$.

Conforme os números da tabela acima, pode-se constatar que, aos poucos, o número de artigos enviados fora do template vem sendo reduzido de forma bastante lenta. Isso pode indicar que os autores ainda encontram dificuldades em se adequar ao novo dispositivo, mesmo que a grande maioria dos periódicos o estejam adotando. Demonstra, também, que a maioria dos artigos devolvidos

9 Os dados utilizados na elaboração da Tabela 1 - Fluxo editorial dos artigos no período de janeiro de 2018 a agosto de 2018 da revista Educação (UFSM) - foram obtidos através do SEER. 
aos referidos autores por terem, em um primeiro momento, enviado suas colaborações fora do dispositivo template, voltaram a serem encaminhados e, nesse segundo momento, de forma correta.

Abaixo, serão demonstrados os gráficos referentes ao questionário realizado junto aos autores e avaliadores ad hoc quanto ao uso obrigatório do template a partir de janeiro de 2018 da revista Educação (UFSM).



Figura 2: RESPOSTA AO QUESTIONÁRIO PARA AUTORES DA REVISTA EDUCAÇÃo (UFSM). FONTE: ELABORADA PELOS AUTORES (2018).

O gráfico acima (Figura 2) demonstra ser muito boa a aceitação do template por parte dos autores da Revista, em virtude de 92,1\% responderem ser ótimo ou bom, enquanto somente $2,6 \%$ responderam ser regular e 5,3\% acreditarem ser ruim.

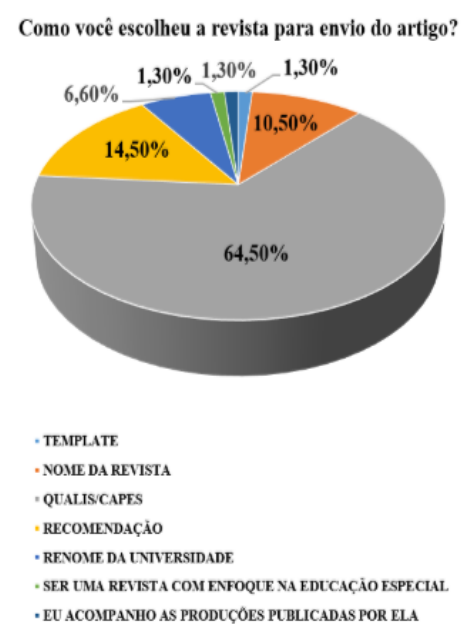

Figura 3: Resposta AO QUESTIONÁRIO para aUtores dA REVISTA EdUCAÇÃo (UFSM). FONTE: ELABORADA PELOS AUTORES (2018). 
Conforme apontado durante o texto e evidenciado no gráfico acima (Figura 3), a avaliação da revista no Qualis/CAPES colaborou para sua visibilidade, principalmente pois as avaliações A1/A2 e B1 são as qualificações mais perseguidas no cenário da produção científica da Pós-Graduação brasileira ${ }^{10}$. Vale destacar que, nesta pergunta, houve um equívoco, tendo em vista que um usuário confundiu a revista Educação (UFSM) com a Revista Educação Especial, isso provavelmente deve-se ao fato das revistas serem editadas pelo mesmo setor.

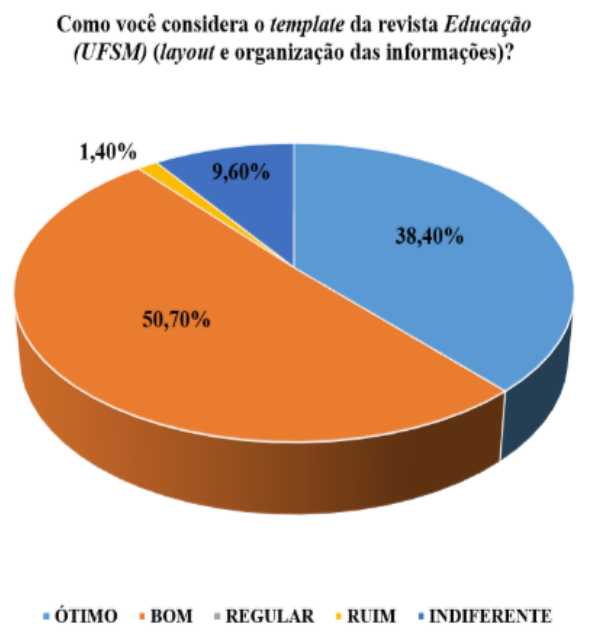

FiguRA 4: RESPOSTA AO QUESTIONÁRIO PARA AVALIADORES AD HOC DA REVISTA EDUCAÇÃO (UFSM). FONTE: ELABORADA PELOS AUTORES (2018).

Analisando o gráfico acima (Figura 4) acima, percebe-se que o percentual de $89,1 \%$ considerou o layout e a organização das informações do template ótimas e boas, constatando que, para os avaliadores da Revista, o template ajudou os autores na organização correta das informações que devem constar em um artigo indexado à Revista. Pode-se melhorar, a fim de que os $11,1 \%$ que acharam ruim ou foram indiferentes ao template mudem seu posicionamento.

Um percentual significativo de 90,4\%, no gráfico abaixo (Figura 5), afirmou que o template agiliza na avaliação dos trabalhos apresentados por parte dos professores. Assim, acredita-se que se deve estar sempre inovando para melhorar o processo de inserção dos manuscritos por parte dos autores junto ao programa SEER na revista Educação (UFSM).

10 Dados obtidos da Plataforma Sucupira da CAPES. Disponível em: https://sucupira.capes.gov. br/sucupira/public/consultas/coleta/veiculoPublicacaoQualis/listaConsultaGeralPeriodicos.jsf. Acesso em: 20 jun. 2018. 


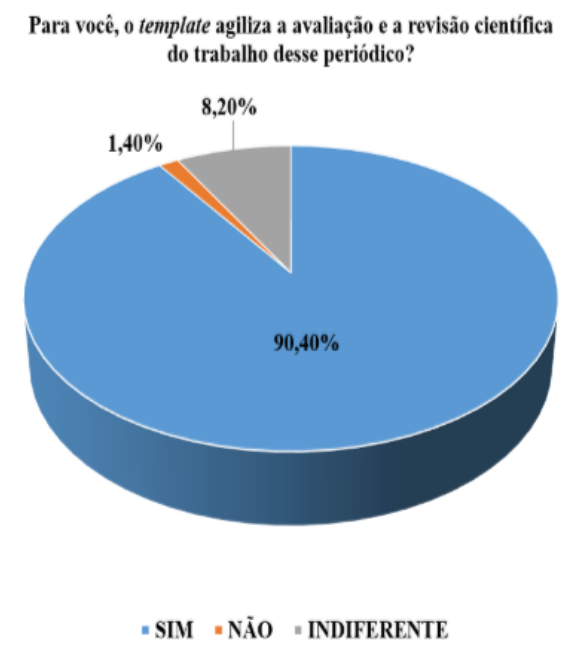

Figura 5: RESPOSTA AO QUESTIONÁRIO PARA AVALIADORES AD HOC DA REVISTA EDUCAÇÃO (UFSM). FONTE: ELABORADA PELOS AUTORES (2018).

\section{CONSIDERAÇÕES FINAIS}

O template implementado na revista Educação (UFSM) tem demonstrado efeitos positivos, visto que os autores deixaram de enviar seus trabalhos fora das normas, o que, anteriormente, ocasionava uma devolutiva em grande número. É notória a melhora nesse sentido e, consequentemente, principalmente, a menor dificuldade na execução dos trabalhos editoriais. Assim, pode-se afirmar que melhorou a editoração do periódico, uma vez que não se faz mais necessário refazer a toda padronização dos textos para a edição final. Entende-se a Revista como um dos principais recursos para a divulgação, compartilhamento da comunicação e informação e, também, popularização da ciência e do saber produzido nas universidades.

A contribuição do experimento prático do trabalho está no aprimoramento e no envolvimento de todos os atores do processo editorial, que se faz necessário para se chegar a uma produção científica através do trabalho colaborativo e participativo de pessoas localizadas em diversas partes geográficas do mundo, um fazer que só as tecnologias em rede podem proporcionar, trazendo benefícios à sociedade.

Finalizando-se a pesquisa, acredita-se ter alcançado o objetivo proposto de criar um produto que viesse ao encontro do que o setor tem produzido: um dispositivo facilitador, prático e de fácil acesso aos autores de artigos, disponibilizado 
à comunidade para uso obrigatório no começo do ano de 2018. O formulário demonstra de forma prática o que cada seção do artigo deve conter, com o propósito de facilitar a interpretação por parte dos usuários do sistema eletrônico para coleta dos trabalhos ao periódico, de forma transparente e que possa servir a todos os envolvidos no processo de produção de conteúdo científico. O referido dispositivo mostrou eficiência aos usuários, pois, além das facilidades, proporciona uma maior segurança aos autores que enviam seus artigos de forma correta. O dispositivo respalda a credibilidade conquistada pelo periódico estudado, que sempre teve a preocupação de oferecer aos autores um instrumento de padronização, garantindo maior segurança aos autores na hora de postar o seu manuscrito no sistema e, com isso, agilizar a vida dos autores como também as publicações, após aprovação do artigo, até o momento da edição.

Nesse sentido, reitera-se que é necessário promover uma construção coletiva e reflexão crítica, explicitamente consciente e racional com os agentes envolvidos na gestão de revistas científicas acadêmicas, para aprimorar o sistema de apresentação do conhecimento do periódico estudado. Sejam eles docentes, técnicos administrativos ou acadêmicos envolvidos na produção editorial do conteúdo científico para a área da Educação, o qual espera-se que as suas vivências e práticas administrativas possam contribuir para potencializar e transformar o almejado crescimento e reconhecimento do periódico, alicerçado em portais institucionais.

\section{REFERÊNCIAS}

MAIA, Luiz Cláudio Gomes. Um estudo sobre o uso de periódicos eletrônicos: O Portal de Periódicos CAPES na Universidade Federal de Minas Gerais. 2005. 153 p. Dissertação (Mestrado em Ciência da Informação) - Universidade Federal de Minas Gerais, Belo Horizonte, 2005.

OLIVEIRA, Érica Beatriz Pinto Moreschi de. Periódicos científicos eletrônicos: definições e histórico. Inf. \& Soc.: Est., João Pessoa, v. 18, n. 2, p. 69-77, maio/ago. 2008. Disponível em: http://www.brapci.inf.br/index.php/article/ download/12349. Acesso em: 04 abr. 2018.

VALERIO, Palmira Maria Caminha Moriconi. Periódicos científicos eletrônicos e novas perspectivas de comunicação e divulgação para a ciência. 2005. 210 p. Tese (Doutorado em Ciência da Informação) - Universidade Federal do Rio de Janeiro, Rio de Janeiro, 2005. 